\title{
Exploring Machine learning application in exhibition layout of museum
}

\author{
Lin Pey Fan ${ }^{1}$ and Tzu How Chu ${ }^{2}$ \\ ${ }^{1}$ PhD Candidate, Department of Geography, National Taiwan University, Taiwan \\ ${ }^{2}$ Professor, Department of Geography, National Taiwan University, Taiwan
}

\begin{abstract}
.
Continual revisions and enhancements to the presentation in museum will allow visitors engagement to remain high interest and acquire visiting benefits when interaction within the display objects. The layout task of objects in exhibition gallery of museum is quite complex, high-cost, time-consuming, and laborious manual process. It is essential and necessary to establish a customized recommendation scheme of exhibition spatial layouts to provide museum crews the configuration frameworks of gallery to improve the efficient of exhibition layout. According to the interactive experience model in museums, we suggest three dimensions: the visitors' behavior, the role of objects, and the layout of space, will benefit to looking for affective and embodied procedures and physical principles of exhibition layout. On the other hand, the state-of-the-art machine learning of artificial intelligence has been widely applied in lots of professional fields (e.g. diagnosis, monitory, prediction, classification, interpretation, scheduling). According to the attributions of exhibition layout and the characteristic of machine learning methods, we suggest that machine learning is a great potential and powerful approach to build up a customized recommendation scheme of exhibition layouts based on the previous knowledge of layout, and it is worth to develop and implement in future research.
\end{abstract}

Keywords: Machine Learning, Exhibition Layout, Physical Principle, Layout Pattern

\section{Introduction and Motivation}

"Millions upon millions of people visit museums" (Ambrose \& Paine, 2018), and the museum is one of the most important cultural attractors (Keng, 2017; Kervankiran, et al., 2016). There is about 70 percent of international tourists participate in culture tourism (Kervankiran et al., 2016). Contacting with the local culture and heritage, tourists like to visit museums (Jolliffe \& Smith, 2001). Museums increasingly look to a general public audience for support, and competition for a market share of people's leisure time is a driving force that focuses the heat on exhibitions (Mclean, 1999). In order to attract more and more tourists to visit museums, curators are making changes to the display of exhibitions more frequently than before (Keng, 2017; Mclean, 1996). 
However, the planning tasks of the exhibition layout are high-cost, time-consuming, and laborious manual process. Besides, these studies or projects of exhibition layout are usually outsourced and carried out by different research or private institutions, and the documents of the layouts are seldom recorded or stored in museums. Therefore, the museum curators would have hardly any chance to accumulate the knowledge or experiences in exhibition planning. Is there a good method or technology to improve the efficiency of the exhibition planning, and to enhance the flexibility of the alternative layout plans? Mclean (1996) proposed that physical spatial layout could be mediated by interactive computer technologies. But what kind of the computer technologies can help museum curators or professors to accumulate the knowledge and experience in physical spatial layout planning?

The knowledge of the exhibition layout is usually stored as texts, which is scattered in literature and it is difficultly for people to acquire. The type of these texts is knowledge-based or rule-based, not data-driven, so acquiring the knowledge of the exhibition layout is big challenge. In 1990s, people proposed the expert system (ES) would be an appropriate method to leverage within spatial planning (Kim et al., 1990). Wagner (2017) viewed hundreds of papers of ES cases studies from 1984 to 2016, and stated that ES applications in planning and design were mainly focus on theories or concepts between 1984 and 1995. There is hardly any research applying the ES to enhance or implement the automation of spatial planning, e.g. urban planning, landuse planning, or site planning. Owing to drawbacks of expert support system, such as extensibility and well-understood, many studies (Levy-Fix et al., 2019; Ling et al., 2017; Ravuri et al., 2018) applied machine learning (ML) models to improve the shortcomings of ES.

There are some researches applying machine learning to museum applications (Majd \& Safabakhsh, 2017). As we know, there is no discuss the application in exhibition spatial layout with machine learning in literatures so far. According to the researches of Levy-Fix et al. (2019), Ling et al. (2017), and Ravuri et al. (2018), we suggest that combining the configuration concepts of expert system with the ML technologies can provide good solutions to spatial planning or designs.

The objective of this article is exploring the potential approaches of machine learning for exhibition spatial layout. The article contains five main parts. The premier one is introducing the general problems and proposing solution for the topic. The rest of this article is structured as follows. We present some related literature about the machine learning and applications of museum in section 2. In section 3, we explore the knowledge of exhibition layout in museums. Finally, the research is concluded in brief and outline the architecture of future researches in section 4.

\section{Machine learning and exhibition layout}

ML, one of the most popular technologies of Artificial intelligence (AI). There are many applications where ML can be of great help in museums (Majd \& Safabakhsh, 2017). Utilizing ML to achieve automatic goals has become the current trend (Zappone et al., 2019). 


\title{
$9^{\text {th }}$ International Conference on Research in SCIENCE \& TECHNOLOGY
}

\author{
20-22 March, 2020 \\ BERLIN,GERMANY
}

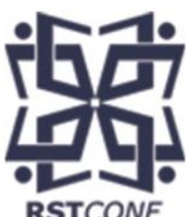

\subsection{Machine learning in museums}

Nowadays, new technologies: Internet of Things (IoT), ML, Virtual Reality (VR), wearable devices, have been applied in museums widely. For commercial and research investigations, it is an important trend to develop technology support museum systems such as multimedia guide by handheld or wearable devices, art authentication and recommendation system according to customer's preference. AI, sometimes called machine intelligence, which has been seeping into our lives, and we use it to accomplish fundamental tasks (Styx, 2019). AI has been discussed in museums for some time (ibid). In 2017, Majd and Safabakhsh attempted to group ML applications in museums into six types: (1) Art authentication, (2) Commercial recommendations, (3) Guiding, (4) 3D virtual reality, (5) Data analysis. (6) Ticketing and museum layout.

First, in order to art authentication, many researchers utilized multi-resolution analysis e.g. wavelets (Polatkan et al., 2009) and filters (Paul et al., 2016; Taylor et al., 2007), to analyze the images of the art works. A statistical model of an artist was built by the scans of a set of authenticated works to prevent art forgery (Johnson et al., 2008). Second, there are many of museums all over the world, and each museum has abundant items of collections. From the aspects of visitors, it is important to find best museums to visit. According to the social networks and personal interests of visitors, commercial recommender systems will provide good solutions to prevent information overload problems (Wang et al., 2007). Third, there are four major kinds of museum tours: human-guided tours, audio tours, online/ virtual tours, and multimedia tours (Majd \& Safabakhsh, 2017). Several researches applied new technologies: Wi-Fi, GPS (Goble Position system), RFID (radio-frequency identification)/ NFC (near-field communication), and Bluetooth to aware of the visitor's location. AI techniques are engaged to auto-guided visitors in museums (Anwer et al., 2016; Cheng et al., 2018).

Forth, VR is an effective communication medium in the museum field. In 2018, Spriporn and Tsai applied the VR technology and support vector machine classification technique to predict tourists' behavior. For educational purposes in museums, Barkova et al. (2018) reviewed new technologies: Interactivity, VR, and ML in games-based scenarios in non-leisure contexts for educational purposes in museums. Fifth, the data analysis is an important issue to identify the visitor motivations and satisfactions in museums. Miguel et al. (2018) integrated data sources to generate and recommend itineraries for museums. Some studies (Bagdanov et al., 2012; Wang et al., 2007) to inspect visitors' preferences in museums for establishing recommendations. Finally, from the aspect of ticketing and museum layout, there are two important issues: the prediction of visitors flow track and the prevention of crowded visits (Majd \& Safabakhsh, 2017). The machine learning application in ticketing and museum layout is to analyze the visitors' behavior in the already existing exhibition space.

From forementioned applications in museum, the ML has great potential and flexible ability to leverage the planning of exhibition layout, and related people will get benefits, e.g. visiting benefits, efficiency of planning task. 


\section{0-22 March, $2020 \quad$ BERLIN,GERMANY}

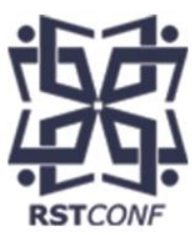

\subsection{Bringing machine learning into exhibition layout}

Traditionally, planners or designers brain storming in workshop many times for several months. During this period, planners discuss, write down their idea, draw up their drafts and modify plans in papers or by the aid of AutoCAD in computer. This process is repeated again and again, and it is a quit time-consuming, high-cost, and laborious manual task. What is the solution to the forementioned problem that people can use this solution a million times over, without doing it the same way twice? Consequently, it is important to improve the experience of museum crews in exhibition spatial layout with display objects utilizing new technologies such as automating layout (Erculiani et al., 2018), machine learning (Dan \& Phuc, 2018; Lin \& Pan, 2019; Pavlidis, 2018; Selin, 2018), big data analysis (Salas-Olmedo et al., 2018), and so on.

ML is algorithms and statistical models that computer systems use to perform a specific task relying on patterns and inference. In 2019, Stetco et al. and Zappone et al. stated that ML models are generally divided into two categories: (1) supervised learning: predicts an output variable using labeled input data, and we distinguish between models that predict a numeric variable or a categorical variable. (2) unsupervised learning: draws inferences from data without labeled inputs such as clustering algorithms and recommender systems. Dan and Phuc (2018) and Stetco et al. (2019) noted that ML uses interconnection of neurons as processing system consisting of multiple input, weight, bias, and the output activation. The link of neurons in the network figures their interaction and each link be associated with weight. Specifically, feed-forward neural network is the simplest and effective type of the artificial neural networks (ANNs), implements the external data into one or several hidden layers and transforms them into the output.

Deep learning is part of machine learning methods based on ANNs, which has multiple layers between the input and output layers (Zappone et al., 2019; Stetco et al., 2019). Deep learning architectures such as deep neural networks, deep belief networks, recurrent neural networks (RNNs) and convolutional neural networks (CNNs) have been applied to many fields. The data in RNNs can flow in any direction, and RNNs are used for applications such as language modeling, and long short-term memory. The CNNs are utilized in computer vision, automatic speech recognition, spatial data, and so on. Zappone et al. (2019) pointed out that the applications of deep learning to design and optimization offers more possibilities than pure data-driven approach. Machine-learned models can learn and generalize patterns found in datasets.

"Patterns serve as generic guiding principles for design. Each pattern describes a problem which occurs over and over again in our environment" (Dawes \& Ostwald, 2017). Patterns are powerful phenomenological design tools. Consequently, utilzing the ML algorithms to build a mathematical model based on sample datasets, the exhibition spatial layout patterns as "training data", and then to make the layout patterns optimize depending on the different thematic of display objects. The optimization suggests explicitly programmed to perform the exhibition spatial layout as reference to curators or planners. The final implement will be determined and revised according to the creative and innovate of the planners' preference.

In order to establish the layout patterns database for ML training, it is necessary to build up the synthesis layout patterns as database. Fundamentally, the synthesis of the exhibition spatial 


\section{0-22 March, 2020 BERLIN,GERMANY}

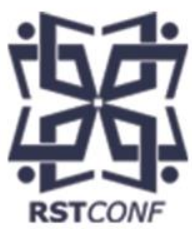

layout patterns should depend on the previous knowledge. The basic concept can be adopted the interactive experience model proposed by Falk and Dierking.

\section{Knowledge of exhibition layout}

The structure of exhibition space layouts has impacted on visitors' movement choices, and visitors tended to make their spatial decisions based on the location of object and the amount of area around that exhibit (Nubani et al., 2018).

\subsection{Exhibition space is core of a museum}

Museum can be classified by different categories such as collections, runners, serve areas, and so on; the subtypes classified by collections can be divided into general museum, archaeology museum, art museum, history museum, science museum, geology museum, industrial museum, and so on (Ambrose \& Paine, 2018). In other words, there are various types of museums, and each type has its characteristic. Basically, some physical principles of exhibition layout could be adjusted depending on the type of museum and the properties of objects, and then the spatial layout patterns database also could be adjusted synchronously for ML during the implement period.

The museum is a multi-layered and a complex environment that represents a diverse set of service units, such as exhibition space, store space, administration space, restaurant space and shop space to match museums' multi-goals (Ambrose \& Paine, 2018). The layouts in the exhibition space are quiet hierarchical: the whole museum arena, the museum building's type, the configure shape of entire exhibition spaces, and the individual exhibition space, where spaces led to other spaces, giving visitors multidirectional views. The layout of exhibition space in this article was focus on the individual exhibition gallery space where each is surrounded with walls, with an entrance and an exit, and where objects are arranged to display.

The exhibition, which interprets and transmits the object, culture, history and environment, and it is a kind of presentation of multiple technologies that implement communications and transmissions of different contents. Exhibition space acts as the basic and main attracted space in museum (Ahmad et al., 2014; Ambrose \& Paine, 2018; Moser, 2010; Li et al., 2013). Demir (2012) and Tzortzi (2015) noted that one of the main functions of a museum is organizing and displaying the selected objects into a meaningful story for visitors. The exhibition space is the core of a museum (Ahmad et al., 2014; Tzortzi, 2015; Mclean, 1996).

Usually, museums do not have enough exhibition spaces to display physically the thousands of objects in one time (Carliner, 2001). The creation of exhibitions has become the province of specialist planners, curators and educators who focus on communicating key messages via a vast array of display strategies (Ambrose \& Paine, 2018; Moser, 2010). Mclean (1999) and Demir (2012) stated that exhibition is a fundamental mission to empower visitors and bridge the gap between the experts and the laymen with objects and experiments. Moser (2010) stated that space layout in exhibition is a basic part of the way displays. The exhibits in museum provide a framework and shape the visitors' perceptions of what is presented (Jolliffe \& Smith, 2001). Basically, exhibition spatial layout should be well considerated base on the visitors' behavior and need. 


\section{0-22 March, 2020 \\ BERLIN,GERMANY}

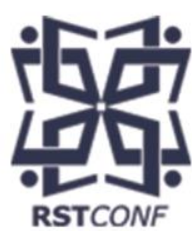

\subsection{Exploring the dimensions of exhibition layout}

Interactive experience model, proposed by Falk and Dierking in 1991, which is dictated to personal context, social context, and physical context, and each of these contexts is continuously constructed by the visitor, and the interaction of these create the visitor's experience (Falk \& Dierking, 2016). This model created a framework for making sense of museum visits and experiences, and we can understand visitors' perspective through by three contexts in museum (Fig. 1):

Figure 1: Interactive experience model

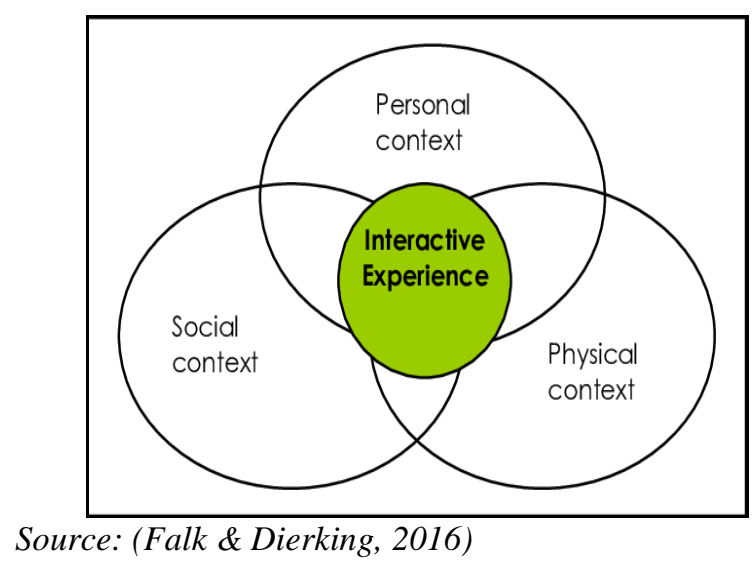

(1) Personal context: it incorporates a variety of experiences and knowledge, including prior knowledge, experience, interests, motivations, concerns and agenda. Differences in personal context should help predict many differences in visitor behavior and learning.

(2) Social context: most people visit museums in a group, and those who visit alone come into contact with other visitors and museum staff. Every visitor's perspective is influenced by social context. Whether or not the museum is crowded also strongly influences the visit experience.

(3) Physical context: it includes the architecture, feel of the building, as well as objects and artifacts. How visitors behave, what they observe, and what they remember are intensely influenced by the physical context.

The exhibition spatial layout is basically to deal with the relationship between visitors and the attributions of space, the relationship between visitors and objects, and the arrangement of objects in space. In other words, exhibition layout should be based on the visitors' behavior and needs, the thematic of display, and the attributions of the objects and space. Consequently, the three contexts of interactive experience model would be divided into two main aspects in the exhibition gallery: people and physical environment. And in order to find out the physical principles for exhibition spatial layout, the aspect of people is the dimension of visitors' behavior about spatial. The aspect of physical environment are subdivided into the role of objects, and the layout of space dimensions.

There are four physical items of visitors' behavior, including amount of visitors, orientation, visiting time, and visual distance. And there are three physical items of the objects, including cluster (or segement), attributions, and buffer area. The physical items in the layout of exhibition space are containment, capacity, centrality, area ratio, and path (aisle) (Tab. 1). 
$9^{\text {th }}$ International Conference on Research in

SCIENCE \& TECHNOLOGY

20-22 March, $2020 \quad$ BERLIN,GERMANY

Table 1: Principles factors raised by interactive experience model

\begin{tabular}{|c|c|c|c|}
\hline Interactive Contexts & Aspects & Dimensions & Physical items \\
\hline Personal Context & \multirow{2}{*}{ People } & \multirow{2}{*}{ Visitors' Behavior } & Amount \\
\hline Social Context & & & $\begin{array}{l}\text { Orientation } \\
\text { Visiting time } \\
\text { Visual distance }\end{array}$ \\
\hline \multirow[t]{2}{*}{ Physical Context } & \multirow[t]{2}{*}{$\begin{array}{c}\text { Physical } \\
\text { Environment }\end{array}$} & Role of Objects & $\begin{array}{l}\text { Cluster/ Segment } \\
\text { Attributions } \\
\text { Buffer area }\end{array}$ \\
\hline & & Layout of Space & $\begin{array}{l}\text { Containment } \\
\text { Capacity } \\
\text { Centrality } \\
\text { Area ratio } \\
\text { Path (Aisle) }\end{array}$ \\
\hline
\end{tabular}

Source: (this study)

\section{Conclusion and future works}

The exhibition layout synthesis in museum refers to the problem of arranging exhibit objects in accordance to design preferences and hard constraints. It encompasses jobs like arranging exhibit displays within a gallery, visitors flow, and planning entire floors. The constraints indicate to encode functional, structural requirements, and exhibit displays features (e.g. size, shape, weight). The future work will be focus on the challenging problem of preserving expert experiences in exhibition layout synthesis with exhibit displays to assist both expert and amateur museum crews in this endeavor, enhancing productivity and outcome quality. And, a recommendation scheme will be developed in synthesis exhibition layouts with display objects to museum crews by heuristic technologies (e.g. Genetic Algorithm, Tabu Algorithm) and machine learning (e.g. Multi-class logistic regression, CNNs, RNNs). We propose the architecture of future works to implement the recommendation scheme in exhibition layout of museum with railcars for example (Fig. 2), and the main works are following:. 
Figure 2: The framework of future works in a recommendation scheme of exhibition layouts

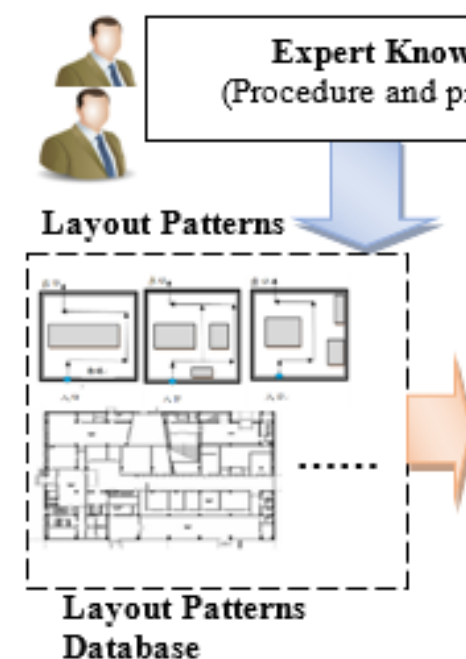

Database

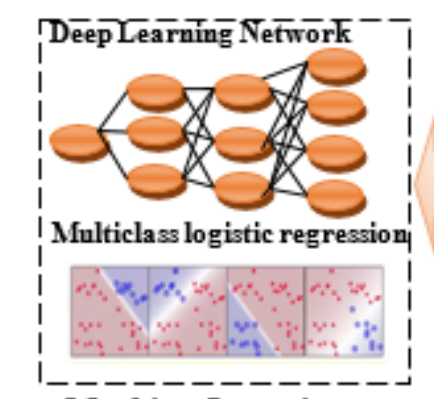

\section{Machine Learning}
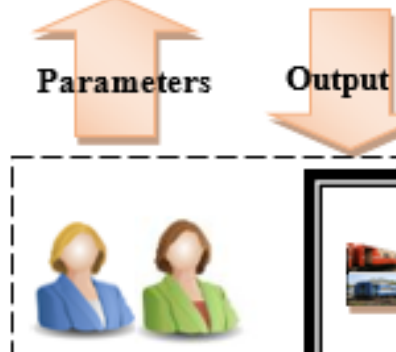

Museum Crews

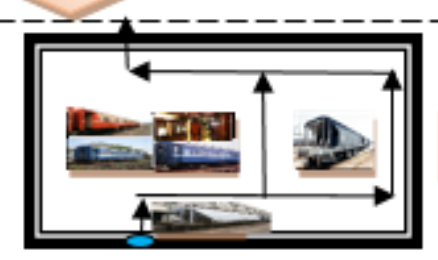

Recommendation of layouts

\section{Collecting objects}

(locomotive, railcar, engineering car...)
Heuristic Technologies (e.g. Genetic Algorithm, Tabu Search)

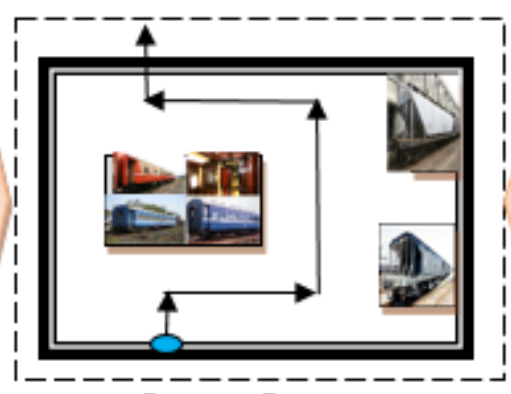

Layout Instance

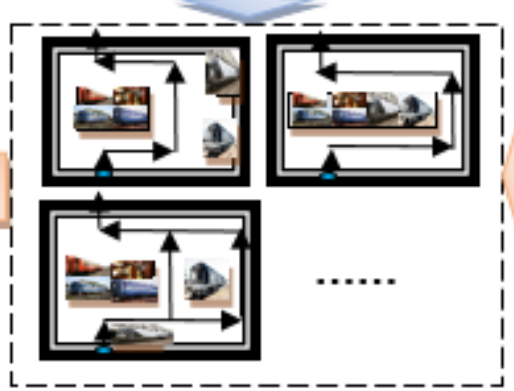

Layouts Instances Database

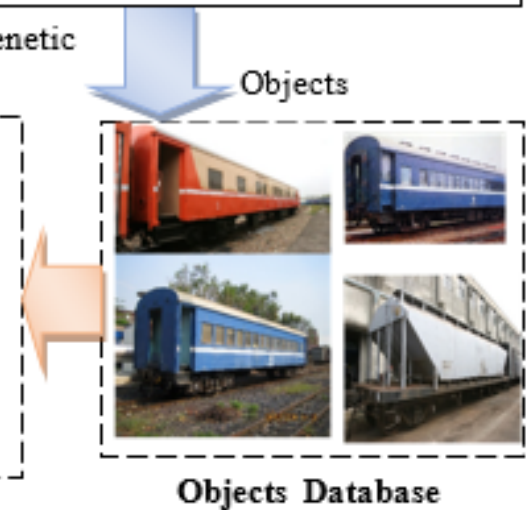

Objects Database

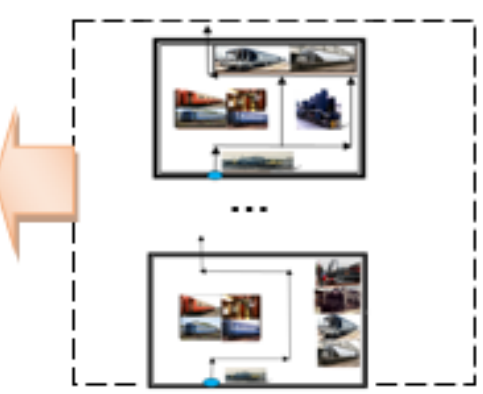

Layouts Instance Database past experiences

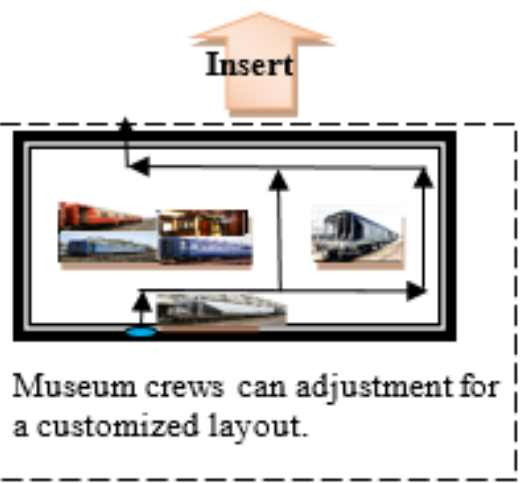

Source: (this study) 
(1) Simulating layout patterns from expert knowledge

People can use methodology of the morphological, the point-line-plane-stereo concept, to integrate the principles and deduce main procedures of exhibition layout based on the three dimensions from previous literatures' findings, suggestions, and results. Those principles will be the array strategies to configure the framework of exhibition gallery, and they can be adjusted depending on the museum types (e.g. transport, industry, art, history), and the characteristics of objects (e.g. plane/ three-dimensions, heavy/ light, big/ small). According to these procedures and principles, people can simulate and synthesis variety layout patterns under different thematic to form the patterns database.

(2) Selecting certain type of museums to mimic with layout patterns

There are various types of museums, and each type has its characteristic. For example, a railway museum belongs to industrial and transport museums. The collections of the railway museum are mainly about railway industry materials, and the properties of those objects, such as locomotives, railcars, and tool machines, which are basically quite huge: high, heavy, lengthy, and wide. Those immense and heavy objects fundamentally need large and spacious exhibition space to display, and they are usually placed on the ground of first floor. So some physical principles of exhibition layout could be adjusted depending on the type of museum and the properties of objects, and then the spatial layout patterns database also could be adjusted synchronously for ML during the experimental period.

(3) Utilizing heuristic genetic algorithm to implement the topic

Future works will be focus on the challenging problem of preserving expert knowledge and experiences in exhibition layout synthesis with display objects to assist both expert and amateur museum crews in this endeavor, enhancing productivity and outcome quality. And, a recommendation system will be developed in synthesis exhibition layouts with exhibit objects to museum crews by heuristic technologies (e.g. Genetic Algorithm, Tabu Algorithm) and machine learning (e.g. Multi-class logistic regression, CNNs, RNNs).

In summary, striking the right balance between exhibition spatial layout constraints and exhibit displays attributes is very difficult. Even experienced expert designers often depend on trial and error. ML has been utilizing in many domains to increase levels of automation and acquire good results. We propose that ML is quite potential and feasible approach for leverage within exhibition layout to achieve the goal of customized recommendtation scheme, which can help people collect and accumulate the knowledge of exhibition spatial layout, provide museum crews the configuration frameworks of gallery, and reduce the cost of planning and the laborious manual process, to achieve and match the need of shortening rearrangement time to attract more people to visit museums. We hope that the task of construcing the customized recommendation scheme in exhibtion layout will be implemented in the near future. 


\section{References}

[1] Ahmad, S., Abbas, M. Y., Taib, M. Z-M. and Masri, M. (2014). Museum Exhibition Design: Communication of meaning and the shaping of knowledge, Procedia-Social and Behavioral Sciences, vol. 153, pp. 254-265.

[2] Anwer, R. M., Khan, F. S., Weijer, J. V. and Laaksonen,J. (2016). Combining holistic and part-based deep Representations for computational painting categorization, 2016 ACM International Conference on Multimedia Retrieval, New York, USA, pp. 339-342.

[3] Ambrose, T. and Paine, C. (2018). Museum Basics (4nd). The International Council of Museum in UNESCO, Routledge, London and New York.

[4] Bagdanov, A. D., Bimbo, A. D., Landucci, L. and Pernici, F. (2012). MNEMOSYNE: Enhancing the museum experience through interactive media and visual profiling, Multimedia for Cultural Heritage, pp. 39-50.

[5] Barkova, O., Pysarevska, N., Allenin, O., Hamotsky, S. Gordienko, N., Sarnatskyi, V., Ovcharenko, V., Tkachenko, M., Gordienko, Y. and Stirenko, S. (2018). Gamification for Education of the Digitally Native Generation by Means of Virtual Reality, Augmented Reality, Machine Learning, and Brain-Computing Interfaces in Museums, Uncommon Culture, vol. 7, no.1/2(13/14), pp. 86-101.

[6] Carliner, S. (2001). Modeling Information for Three-dimensional Space: Lessons Learned from Museum Exhibit Design. Technical Communication, vol. 48(1), pp. 66-81.

[7] Cheng, F. C. Z., Wang, Y. and Chen, J. J. (2018). Integration of Open Source Platform Duckietown and Gesture Recognition as an Interactive Interface for the Museum Robotic Guide. 2018 27th Wireless and Optical Communications Conference, Hualien, Taiwan, pp. $1-5$

[8] Dan, T. X. and Phuc, P. N. K. (2018). Application of machine learning in forecasting energy usage of building design. 2018 4th International Conference on Green Technology and Sustainable Development, Ho Chi Minh City, Vietnam, pp. 53-59.

[9] Dawes, M. J. and Ostwald, M. J. (2017). Christopher Alexander's A Patterns Language: analyzing, mapping and classifying the critical response. City, Territory and Architecture, pp. 4-17.

[10]Demir, C., (2012) Graphic design for a permanent exhibition: exhibition design of the Museum Mimar Kemaleddin, Procedia- Social and Behavioral Sciences, vol. 51, pp. 495-500.

[11]Erculiani, L., Dragone, P., Teso, S. and Passerini, A. (2018). Automating layout synthesis with constructive preference elicitation, Machine Learning and Knowledge Discovery in Databases, Springer Nature Switzerland AG, Subseries of Lecture Notes in Computer Science 11053, pp. 254-270.

[12]Falk, J. H. and Dierking, L.D. (2016). The Museum Experience. Routledge, New York. 
$9^{\text {th }}$ International Conference on Research in SCIENCE \& TECHNOLOGY

20-22 March, 2020

BERLIN,GERMANY

[13]Levy-Fix, G., Kuperman, J. G. and Elhadad, N. (2019). Machine learning and visualization in clinical decision support: current state and future directions. Arxiv.org. https://arxiv.org/abs/1906.02664

[14] Johnson, C. R., Hendriks, E., Berezhnoy, I. J., Brevdo, E., Hughes, S. M., Daubechies, I., Li, J., Postma, E. and Wang, J. Z. (2008). Image processing for artist identification, IEEE Signal Processing Magazine, vol. 54, pp. 37-48.

[15] Jolliffe, L. and Smith, R., (2001) Heritage, Tourism and Museums: the case of the North Atlantic islands of Skye, Scotland and Prince Edward Island, Canada. International Journal of Heritage Studies, vol. (2), pp. 149-172.

[16]Keng, F. Y. (2017). Breakthrough and Innovation: New Visitors for National Museum Exhibition. Museology Quarterly, vol. 31(1), pp. 79-98.

[17] Kervankiran, I., Temurcin, K. and Yakar, M. (2016). The Development of Museology in Turkey, a Spatial Analysis of Museums and their Contribution to Tourism in Turkey, AlmaTourism, vol. 13, pp. 1-22.

[18] Kim, T.J., Wiggins, L. L. and Wright, J. R. (eds) (1990). Expert systems: Applications to Urban Planning, Springer-Verlag NewYork Inc., USA.

[19]Li, Z., Wei, Q. and He, H. (2013). A brief analysis of spatial constitution and functional organization of museum architecture: A case study on museums in Hefei, Frontiers of Architectural Research, vol. 2, pp. 354-361.

[20]Lin, Y. and Pan, D. Z. (2019). Machine learning in physical verification, mask synthesis, and physical design. In: Elfadel I., Boning D., Li X. (eds) Machine Learning in VLSI Computer-Aided Design. Springer, Cham. pp. 95-115.

[21]Ling, Y. Hasan, S. A., Datla, V. Qadir, A., Lee, K., Liu, J., and Farri, O. (2017). Diagnostic inferencing via improving clinical concept extraction with deep reinforcement learning: a preliminary study. 2017 Machine Learning for Healthcare Conference, Boston, USA, pp. 271-285.

[22] Macdonald, S. (2007). Interconnecting: museum visiting and exhibition design, CoDesign, vol. 3(S1), pp. 149-162.

[23] Majd, M. and Safabakhsh, R. (2017). Impact of machine learning on improvement of user experience in museums, Artificial Intelligence and Signal Processing (AISP), Shiraz, Iran, pp. 195-200.

[24] Mclean, K. (1996). Planning for People in Museum Exhibition, Association of Science Technology Centers, Washington, DC, USA.

[25] McLean, K. (1999). Museum Exhibitions and the Dynamics of Dialogue, Daedalus, vol. 128(3), America's Museums (1999), pp: 83-107. The MIT Press. http://www.jstor.org/stable/20027568 
[26] Miguel, T. R., Felix, M., Roberto, Z., Giovanni, G., Rolando, Q. and Marco, M. I. (2018). A recommender system to generate museum itineraries applying augmented reality and social-sensor mining techniques, Virtual Reality, pp.1-15.

[27]Moser, S. (2010). The Devil is in the Detail: Museum Displays and the Creation of Knowledge, Museum Anthropology, vol. 33(1), pp. 22-32.

[28] Nubani, L., Puryear, A. and Kellom, K. (2018) Measuring the Effect of Visual Exposure and Saliency of Museum Exhibits on Visitors' Level of Contact and Engagement, Behavioral Science, vol. 8 (100), pp. 1-14.

[29]Paul, B., Irina, C., Manuel, D. G., Felix, J. X. and Marios, S. (2016). Simultaneous forgery identification and localization in paintings using advanced correlation filters. 2016 IEEE International Conference on Image Processing, Arizona, USA, pp. 146-150.

[30]Polatkan, G., Jafarpour, S., Brasoveanu, A., Hughes, S. and Daubechies, I. (2009). Detection of forgery in paintings using supervised learning. 16th IEEE International Conference on Image Processing, Cairo, Egypt, pp. 2921-2924.

[31] Pavlidis, G. (2018). Apollo - A hybrid recommender for museums and cultural tourism. 2018 International Conference on Intelligent Systems, Madeira, Portugal pp. 94-101.

[32] Ravuri, M., Kannan, A., Tso, G. J. and Amatriain, X. (2018) Learning from the experts: From expert systems to machine-learned diagnosis models. Proceedings of Machine Learning Research, vol. 85, pp. 1-16.

[33] Salas-Olmedo, M. H., Moya-Gomez, M., Garcia-Palomares, J. C., and Gutierrez, J. (2018). Tourists' digital footprint in cities: Comparing big data sources, Tourism Management, vol. 66, pp 13-25.

[34] Selin, J. and Rossi, M. (2018). The functional design method for buildings (FDM) with gamification of information models and AI help to design safer buildings. 2018 Federated Conference on Computer Science and Information Systems 15. Poznań, Poland, pp. 907-911.

[35] Spriporn, K. and Tsai, C. F. (2018). Predicting tourists' behavior of virtual museum using support vector machine with feature selection technique. 2018 International Conference on Machine Learning and Cybernetics, Chengdu, China, pp. 443-438.

[36] Stetco, A., Dinmohammadi, F., Zhao, X., Robu, V., Flynn, D. Barnes, M., Keane, J., and Nenadic, G. (2019). Machine learning methods for wind turbine condition monitoring: A review, Renewable Energy, vol. 133(C), pp. 620-635.

[37] Styx, L. (2019). How are museums using artificial intelligence, and is AI the future of museums? MuseumNext [Online]. https://www.museumnext.com/article/artificial-intelligence-and-the-future-of-museums/

[38] Taylor, R. P., Guzman, R., Martin, T. P., Hall, G. D. R., Micolich, A. P., Jonas, D., Scannell, B. C., Fairbanks, M. S. and Marlow, C. A. (2007). Authenticating Pollock paintings using fractal geometry, Pattern Recognition Letters, vol. 28, pp. 695-702. 


\section{$9^{\text {th }}$ International Conference on Research in SCIENCE \& TECHNOLOGY \\ 20-22 March, 2020

[39] Tzortzi, K. (2015). Spatial concepts in museum theory and practice. 10th International Space Syntax Symposium, London, UK, pp. (37) 1-16.

[40]Wang, Y., Aroyo, L. M., Stash, N. and Rutledge, L. (2007). Interactive user modeling for personalized access to museum collections: the Rijksmuseum case study. International Conference on User Modeling, Corfu, Greece, pp. 385-389.

[41]Wagner, W.P. (2017). Trends in expert system development: A longitudinal content analysis of over thirty years of expert system case studies. Expert Systems With Applications, vol. 76, pp. 85-96.

[42]Zappone, A., Renzo, M. D., Debbah, M., Lam, T. T. and Qian, X. (2019). Model-Aided Wireless Artificial Intelligence: Embedding Expert Knowledge in Deep Neural Networks Towards Wireless Systems Optimization, IEEE Vehicular Technology Magazine, pp. 1-7. 